\title{
Estimating the Monetary Policy Measures of Japan in Shadow/ZLB Term Structure Model
}

\author{
Rui WANG ${ }^{1}$ \\ ${ }^{1}$ Associated Professor at Department of Economics, Kanto Gakuen University. Research Fellow at Graduate School of \\ Economics, Kobe University, Japan \\ Correspondence: Rui WANG, 200 Fujiagu-cho, Ota, Gunma Prefecture, 373-0034, Japan.
}

Received: August 13, 2019

Accepted: September 2, $2019 \quad$ Available online: October 28, 2019

doi:10.11114/aef.v6i6.4577

URL: https://doi.org/10.11114/aef.v6i6.4577

\begin{abstract}
In this paper, we follow the estimation methodology proposed by Krippner (2015) and use Japanese government bond yield curve data to estimate a shadow/ZLB term structure model. This model provides three estimated monetary policy measures, SSR, ETZ and EMS, which can be used to gauge the stance of monetary in a consistent way in both ZLB and non-ZLB environment. Japan has experienced a long period of the ZLB since 1999. The policy rate has already lost its function as an appropriate quantitative measure of monetary policy. The SSR estimated from the shadow/ZLB term structure model can evolve to negative level in the ZLB environment and provide consistent view of the stance of monetary policy as the positive short policy interest rate dose in the normal non-ZLB environment. The ETZ answers the question that how long the short interest rate will be expected to be restricted by the ZLB, which can be useful for the central bank as a reference for exit strategy of unconventional monetary easing or forward guidance on public expectation formation. The EMS measures the stance of monetary policy, relatively tight or relatively loose, in a consistent and comparable way under both ZLB and non-ZLB environment. The analysis shows that all three measures exhibit very good traceability of monetary policy in Japan, which can also be used as the proxy variables for the stance of monetary policy in other econometric procedures for policy evaluation.
\end{abstract}

Keywords: ZLB, GATSM, K-ANSM, monetary policy measures

JEL Classification: E53, E52, G12

\section{Introduction}

Before the Global Financial Crisis (henceforward GFC) since 2008, nominal short interest rates generally evolved above the Zero Lower Bound (henceforward ZLB) and the central banks in major developed countries chose short interest rate, which is also known as policy rate such as Federal Funds Rate (FFR) in US, as the direct operating target of the monetary policy. But after the GFC, central banks in major countries, expect China, have to face the untraditional challenges from the ZLB constraint. The conventional monetary policy, for example, the most typical one is the adjustment of short policy rate, is not practical and operative anymore in the ZLB environment and unconventional monetary policies, such as large scale of asset purchases of government bonds and forward guidance on public expectation formation, have been adopted to provide further stimulus to economy, stopping recession and stabilizing financial system.

In the non-ZLB environment assumed by the orthodox monetary macroeconomic theory, short interest rate is a useful measure for monitoring the stance of monetary policy. This is true for the most of central banks in developed countries that choose the short interest rate as the operating target of monetary policy. The practice of monetary policy in the central banks of major developed countries also have provided this point. But facing the ZLB constraint, short interest rate evolves near the neighborhood of ZLB and it can't provide useful information about the stance of monetary policy. Gaussian Affine Term Structure Model (henceforward GATSM) which is a widely used methodology to fit the term structure of yield curve can provide good description of the dynamics of short interest rates in many macro-financial applications, but when short interest rates are near the ZLB, the performance of GATSM is deficient and unable to provide satisfied fitness of data, just like DSGE modeling, another standard methodology in macroeconomics. Also, in the ZLB environment, the short interest rates are "sticky" which means that they tend to keep approximately static around the ZLB with lower volatilities for a long period of time. This phenomenon can be visually confirmed by Figure 
1 and Figure 2 in Section 3.1, which show that the short interest rates in Japan have experienced along period of the ZLB since 1999. General GATSMs are unable to accommodate this kind of stickiness. In the general GATSM framework, interest rates can evolve below the ZLB to take negative values implicitly, but it is inconsistent with the actual observed data and has less economic implications for practical purpose.

In the ZLB environment, the economic agents generally choose to hold currency and receive zero return actually rather than accept a negative interest rate. This provides the intuition of how to solve the issue of negative interest rates in general GATSM framework. Shadow/ZLB-GATSM proposed by Krippner (2015) is an extension of a general GATSM with the specification of Arbitrage-free Nelson and Siegel (1987) Model (hereafter ANSM). The resulting shadow/ZLB-GATSM framework is also known as Krippner-ANSM (hereafter K-ANSM). Shadow/ZLB-GATSMs represent the shadow term structure which is the term structure that would exist if the currencies were not available. If short interest rates are not restricted by the ZLB and evolve above the ZLB, shadow/ZLB-GATSMs provide same results as general GATSMs do. But shadow/ZLB-GATSMs can provide an adjustment to ensure that interest rates are constrained by the ZLB explicitly. According to Krippner (2015), this adjustment is effectively the optionality form the availability of physical currency as an alternative to the negative interest rates below the ZLB ${ }^{1}$. Another important reason is that the shadow/ZLB-GATSM can provide some useful indicators, Shadow Short Interest Rate (SSR), Expected Time to Zero (ETZ) and Effective Monetary Stimulus (EMS) which can show the stance of monetary policy in the ZLB environment. These indicators can be used as the measures of monetary policy instead of short policy rate because the short policy rate has already lost its effectiveness in the ZLB environment. There are several similar works in this field. Imakubo and Nakajima (2015) estimates a shadow rate term structure model for Japan economy and uses it to extract inflation risk premia from nominal and real term structures. They show that shadow rate term structure model can successfully avoid the estimation bias inherent in the general affine term structure model that ignores the ZLB. Kim and Singleton (2012) investigates the yield curve of Japanese government bond and finds that the shadow rate term structure model can imply risk premiums that can track realized excess returns during extended periods of near-zero short rates in Japan. The main difference of this paper compared to the existing research is that we can extract the policy measures such as ETZ and EMS and use these measures as the reference of monetary policy analysis.

Given this background, we estimated a shadow/ZLB-GATSM for Japan and used the estimation results to evaluate the stance of monetary policy in Japan. The yield curve data set is from 1992/7/10 to 2016/11/24. However, since 2016/2/1, the yield curve data from 3-months maturity to 10-years maturity begun to take negative values due to the start of QQE with a negative interest rate policy, which made data inapplicable to the computation of model estimation. Based on this reason, we excluded the data from 2016/02/01 to 2016/11/24. The remaining of this paper is organized as follows. We specify a K-ANSM(2) model as an implementation of shadow/ZLB-GATSM with detailed description in Section 2 . In Section 3, we talk about the data and estimation and then give the results. In Section 4, we use the main results, three measures of monetary policy to evaluate the stance of monetary policy in Japan. We also check the relationships between these measures and other macroeconomic variables. The Section 5 concludes this paper.

\section{Shadow/ZLB Term Structure Model}

We firstly present a general GATSM with two factors (state variables), level component $L_{t}$ and slope component $S_{t}$ with the same factor loading in the style of Arbitrage-free Nelson and Siegel (1987) model which is widely used for interest rate modeling, hereafter abbreviated to ANSM(2). Then we extend ANSM(2) to adapt to the ZLB environment by incorporating explicit zero lower bound restriction for short-term interest rate in a mathematically consistent fashion, which results a K-ANSM(2) model.

Short-term interest rate $r_{t}$ can be represented as a linear combination of unobservable state variables, $L_{t}$ and $S_{t}$.

$$
r_{t}=c^{\top} x_{t}=\left[\begin{array}{ll}
1 & 1
\end{array}\right]\left[\begin{array}{l}
L_{t} \\
S_{t}
\end{array}\right]=L_{t}+S_{t}
$$

$c^{\top}=\left[\begin{array}{ll}1 & 1\end{array}\right]$ is the factor loading vector. The state variables vector $x_{t}^{\top}=\left[\begin{array}{ll}L_{t} & S_{t}\end{array}\right]$ under the objective physical $\mathbb{P}$ measure evolves as a correlated vector Ornstein-Uhlenbeck stochastic process

$$
d x_{t}=\kappa^{\mathbb{P}}\left(\theta^{\mathbb{P}}-x_{t}\right) d t+\sigma d W_{t}^{\mathbb{P}}
$$

where $d W_{t}^{\mathbb{P}}$ is a $2 \times 1$ vector of independent Wiener increments with each component of $d W_{t}^{\mathbb{P}}$ follows $\mathbb{N}(0,1) \sqrt{d t}$. $\mathbb{N}(0,1)$ is the standard normal distribution. $\theta^{\mathbb{P}}$ is a $2 \times 1$ constant vector which represents the mean level of $x_{t}$ in long-run. $\kappa^{\mathbb{P}}$ is a $2 \times 2$ constant parameter matrix that controls the deterministic mean reversion of $x_{t}$ to its long-run mean level $\theta^{\mathbb{P}} . \sigma$ is a $2 \times 2$

\footnotetext{
${ }^{1}$ For detailed discussions, please refer to Krippner (2015), p44.
} 
constant variance-covariance matrix $\left[\begin{array}{cc}\sigma_{1} & 0 \\ \rho_{12} \sigma_{2} & \sigma_{2} \sqrt{1-\rho_{12}^{2}}\end{array}\right]$ of innovations to $x_{t}$. Note that $\sigma \sigma^{\top}=\left[\begin{array}{cc}\sigma_{1}^{2} & \rho_{12} \sigma_{1} \sigma_{2} \\ \rho_{12} \sigma_{1} \sigma_{2} & \sigma_{2}^{2}\end{array}\right]$

which means that the two disturbance terms in vector stochastic process $x_{t}$ have covariance $\rho_{12} \sigma_{1} \sigma_{2}$. The dynamics of $x_{t}$ under objective $\mathbb{P}$ measure can be solved using Ito's lemma.

$$
x_{t+\tau}=\theta^{\mathbb{P}}+e^{-\kappa^{\mathbb{P}} \tau}\left(x_{t}-\theta^{\mathbb{P}}\right)+\int_{t}^{t+\tau} e^{-\kappa^{\mathbb{P}}(\tau-u)} \sigma d W_{u}^{\mathbb{P}}
$$

In general GATSMs, the market prices of risk $\Pi_{t}$ are typically specified as a linear function of the state variables $x_{t}$, which allows the market prices of risk to vary over time,

$$
\Pi_{t}=\sigma^{-1}\left(\gamma+\Gamma x_{t}\right)
$$

where $\gamma$ is the constant component of the market prices of risks and $\Gamma$ determines the variations in market prices of risks with respect to the state variables $x_{t}$. Since the bonds or securities in financial markets are priced under the risk-adjusted $\mathbb{Q}$ measure, the process of state variables $x_{t}$ must be adjusted to represent the observed term structure of interest rates by modification of parameters. Under the risk-adjusted $\mathbb{Q}$ measure, $x_{t}$ is analogous to its counterpart under $\mathbb{P}$ measure,

$$
d x_{t}=\kappa^{\mathbb{Q}}\left(\theta^{\mathbb{Q}}-x_{t}\right) d t+\sigma d W_{t}^{\mathbb{Q}}
$$

where $\kappa^{\mathbb{Q}}=\kappa^{\mathbb{P}}+\Gamma, \theta^{\mathbb{Q}}=\left(\kappa^{\mathbb{Q}}\right)^{-1}\left(\kappa^{\mathbb{P}} \theta^{\mathbb{P}}-\gamma\right)$ and $d W_{t}^{\mathbb{Q}}=d W_{t}^{\mathbb{P}}+\Pi_{t} d t$. Solving $x_{t}$ under the risk-adjusted $\mathbb{Q}$ measure leads to the expression of $x_{t+\tau}$.

$$
x_{t+\tau}=\theta^{\mathbb{Q}}+e^{-\kappa^{\mathbb{Q}} \tau}\left(x_{t}-\theta^{\mathbb{Q}}\right)+\int_{t}^{t+\tau} e^{-\kappa^{\mathbb{Q}}(\tau-u)} \sigma d W_{u}^{\mathbb{Q}}
$$

Then the expectation of $x_{t+\tau}$ is $\mathbb{E}_{t}^{\mathbb{Q}}\left[x_{t+\tau} \mid x_{t}\right]=\theta^{\mathbb{Q}}+e^{-\kappa^{\mathbb{Q}} \tau}\left(x_{t}-\theta^{\mathbb{Q}}\right)$. With $\operatorname{ANSM}(2)$ specification, $\kappa^{\mathbb{Q}}=\left[\begin{array}{ll}0 & 0 \\ 0 & \varphi\end{array}\right]$ and $\theta^{\mathbb{Q}}=\left[\begin{array}{l}0 \\ 0\end{array}\right]$. The expectation and variance of short interest rate $r_{t}$ at time $t+\tau$ are calculated as follows.

$$
\begin{gathered}
\mathbb{E}_{t}^{\mathbb{Q}}\left[r_{t+\tau} \mid x_{t}\right]=c^{\top} \mathbb{E}_{t}^{\mathbb{Q}}\left[x_{t+\tau} \mid x_{t}\right]=\left[\begin{array}{ll}
1 & 1
\end{array}\right] \exp \left(-\left[\begin{array}{ll}
0 & 0 \\
0 & \varphi
\end{array}\right] \tau\right)\left[\begin{array}{l}
L_{t} \\
S_{t}
\end{array}\right]=L_{t}+e^{-\varphi \tau} S_{t} \\
\mathbb{V} \mathbb{R}_{t}^{\mathbb{Q}}\left[r_{t+\tau} \mid x_{t}\right]=\omega_{\tau}^{2}=\int_{0}^{\tau} c^{\top} e^{-\kappa^{\mathbb{Q}} u} \sigma \sigma^{\top} e^{-\left(\kappa^{\mathbb{Q}}\right)^{\top} u} c d u=\sigma_{1}^{2} \tau+\sigma_{2}^{2} \frac{1-e^{-2 \varphi \tau}}{2 \varphi}+2 \rho_{12} \sigma_{1} \sigma_{2} \frac{1-e^{-\varphi \tau}}{\varphi}
\end{gathered}
$$

Volatility effect $V_{\tau}$ captures the influence that volatility in the short interest rate has on expected returns due to Jensen's Inequality which can be calculated using the Heath, Jarrow and Morton (1992) double integral.

$$
\begin{aligned}
V_{\tau} & =\int_{0}^{\tau} c^{\top} e^{-\kappa^{\mathbb{Q}}(\tau-s)} \sigma\left[\sigma^{\top} \int_{s}^{\tau} e^{-\left(\kappa^{\mathbb{Q}}\right)^{\top}(u-s)} c d u\right] d s \\
& =\frac{\sigma_{1}^{2} \tau^{2}}{2}+\frac{\rho_{12} \sigma_{1} \sigma_{2} \tau}{\varphi}\left(1-e^{-\varphi \tau}\right)+\frac{\sigma^{2}}{2 \varphi^{2}}\left(1-2 e^{-\varphi \tau}+e^{-2 \varphi \tau}\right)
\end{aligned}
$$

Forward interest rate $f_{t, \tau}$ is given by the expectation of short interest rate minus volatility effect.

$$
f_{t, \tau}=\mathbb{E}_{t}^{\mathbb{Q}}\left[r_{t+\tau} \mid x_{t}\right]-V_{\tau}=L_{t}+e^{-\varphi \tau} S_{t}-\left[\frac{\sigma_{1}^{2} \tau^{2}}{2}+\frac{\rho_{12} \sigma_{1} \sigma_{2} \tau}{\varphi}\left(1-e^{-\varphi \tau}\right)+\frac{\sigma^{2}}{2 \varphi^{2}}\left(1-2 e^{-\varphi \tau}+e^{-2 \varphi \tau}\right)\right]
$$

Interest rate $R_{t, \tau}$ at time $t$ with maturity $\tau$ can be obtained using the standard term structure relationship in continuous time ${ }^{2}$.

$$
R_{t, \tau}=\frac{1}{\tau} \int_{0}^{\tau} \mathbb{E}_{t}^{\mathbb{Q}}\left[r_{t+\tau} \mid x_{t}\right] d u-\frac{1}{\tau} \int_{0}^{\tau} V_{\tau} d \tau=a_{\tau}+b_{\tau}^{\top} x_{t}
$$

\footnotetext{
${ }^{2} a_{\tau}$ and $b_{\tau}$ are given as follows.$$
a_{\tau}=-\frac{\sigma_{1}^{2} \tau^{2}}{6}-\frac{\sigma_{2}^{2}}{2 \varphi^{2}}\left[1-\frac{1}{2 \varphi \tau} e^{-2 \varphi \tau}+\frac{2}{\varphi \tau} e^{-\varphi \tau}-\frac{3}{2 \varphi \tau}\right]-\frac{\rho_{12} \sigma_{1} \sigma_{2}}{\varphi^{2}}\left[-\frac{1-e^{-\varphi \tau}}{\varphi \tau}+\frac{\varphi \tau}{2}+e^{-\varphi \tau}\right]
$$$$
b_{\tau}=\left[\begin{array}{c}
1 \\
\frac{1}{\varphi \tau}\left(1-e^{-\varphi \tau}\right)
\end{array}\right]
$$ 
From the previous analysis, we can find that short interest rate can be written as a linear function of state variables like $r_{t}=c^{\top} x_{t}$ and the interest rates with other maturities $\tau$ can also be written as a linear function of state variables $x_{t}$, maturity $\tau$ and parameters like $R_{t, \tau}=a_{\tau}+b_{\tau}^{\top} x_{t}$.

We then extend general GATSM by a intuitive modification to adapt GATSM to the ZLB environment ${ }^{3}$. The extension of general GATSM in the ZLB environment is firstly proposed by Krippner (2012), which is known as shadow/ZLB-GATSM framework. In this framework, imposing ZLB restriction can be represented by a max operator.

$$
\underline{\mathrm{r}}_{t}=\max \left\{0, r_{t}\right\}=r_{t}+\max \left\{-r_{t}, 0\right\}
$$

Here $\underline{r}_{t}$ means the actual short interest rate and the $r_{t}$ is the shadow short interest rate. In general situations, $r_{t} \geq 0$, the economic agent invests at the instantaneous interest rate $r_{t}$ and $\underline{\mathrm{r}}_{t}=r_{t}$. But in the ZLB environment, $r_{t}<0$, the economic agent will choose to hold physical currency and obtain zero return actually with $\underline{\mathrm{r}}_{t}=0$. The short interest rate under forward $t+\tau$ risk-adjusted $\mathbb{Q}$ measure follows the normal distribution $r_{t+\tau} \mid x_{t} \sim \mathbb{N}\left(f_{t, \tau}, \omega_{\tau}^{2}\right)$ where $f_{t, \tau}$ and $\omega_{\tau}^{2}$ represent the forward interest rate and its variance, which means that $f_{t, \tau}=\mathbb{E}_{t+\tau}^{\mathbb{Q}}\left(r_{t+\tau} \mid x_{t}\right)$ where $\mathbb{E}_{t+\tau}^{\mathbb{Q}}$ represents the expectation under forward $t+\tau$ risk-adjusted $\mathbb{Q}$ measure. The conditional variance of $r_{t+\tau} \mid x_{t}$ is time-invariant.

$$
\mathbb{V} \mathbb{R}_{t+\tau}^{\mathbb{Q}}\left[r_{t+\tau} \mid x_{t}\right]=\mathbb{V} \mathbb{\mathbb { R } _ { t } ^ { \mathbb { Q } }}\left[r_{t+\tau} \mid x_{t}\right]=\omega_{\tau}^{2}
$$

Given the distribution of $r_{t+\tau} \mid x_{t}$, its probability density function $\mathbf{P D F}\left(r_{t+\tau} \mid x_{t}\right)$ is given as follows.

$$
\mathbf{P D F}\left(r_{t+\tau} \mid x_{t}\right)=\frac{1}{\omega_{\tau} \sqrt{2 \pi}} \exp \left[-\frac{1}{2}\left(\frac{r_{t+\tau}-f_{t, \tau}}{\omega_{\tau}}\right)^{2}\right]
$$

In practice, we allow a non-zero lower bound $r_{L}$ to represent the ZLB which may be a very small number, approximately equal to zero but not zero actually. In our two-factor model, this can be written as follows.

$$
\begin{gathered}
\underline{\mathrm{r}}_{t}=\max \left\{r_{L}, r_{t}\right\}=\max \left\{r_{L}, L_{t}+S_{t}\right\} \\
\underline{\mathrm{r}}_{t+\tau}=\max \left\{r_{L}, r_{t+\tau}\right\}=r_{t+\tau}+\max \left\{r_{L}-r_{t+\tau}, 0\right\}
\end{gathered}
$$

The forward interest rate as the expectation of $\underline{\mathrm{r}}_{t+\tau}$ can be modified as

$$
\underline{\mathrm{f}}_{t, \tau}=\mathbb{E}_{t+\tau}^{\mathbb{Q}}\left[\underline{\mathrm{r}}_{t+\tau} \mid x_{t}\right]=\mathbb{E}_{t+\tau}^{\mathbb{Q}}\left[r_{t+\tau} \mid x_{t}\right]+\mathbb{E}_{t+\tau}^{\mathbb{Q}}\left[\max \left\{r_{L}-r_{t+\tau}, 0\right\} \mid x_{t}\right]=f_{t, \tau}+z_{t, \tau}
$$

and if we set $r_{L}=0$,

$$
\underline{\mathrm{f}}_{t, \tau}=\mathbb{E}_{t+\tau}^{\mathbb{Q}}\left[\underline{\mathrm{r}}_{t+\tau} \mid x_{t}\right]=\mathbb{E}_{t+\tau}^{\mathbb{Q}}\left[r_{t+\tau} \mid x_{t}\right]+\mathbb{E}_{t+\tau}^{\mathbb{Q}}\left[\max \left\{-r_{t+\tau}, 0\right\} \mid x_{t}\right]=f_{t, \tau}+z_{t, \tau}
$$

where $\mathbb{E}_{t+\tau}^{\mathbb{Q}}\left[r_{t+\tau} \mid x_{t}\right]=f_{t, \tau}$ has been obtained in previous analysis. We now evaluate another part $z_{t, \tau}$ in forward interest rate $\underline{\mathrm{f}}_{t, \tau}$.

$$
\begin{aligned}
z_{t, \tau} & =\mathbb{E}_{t+\tau}^{\mathbb{Q}}\left[\max \left\{r_{L}-r_{t+\tau}, 0\right\} \mid x_{t}\right]=\int_{-\infty}^{\infty} \max \left\{r_{L}-r_{t+\tau}, 0\right\} \cdot \mathbf{P D F}\left(r_{t+\tau} \mid x_{t}\right) d r_{t+\tau} \\
& =\int_{-\infty}^{r_{L}}\left(r_{L}-f_{t, \tau}\right) \frac{1}{\omega_{\tau} \sqrt{2 \pi}} \exp \left[-\frac{1}{2}\left(\frac{r_{t+\tau}-f_{t, \tau}}{\omega_{\tau}}\right)^{2}\right] d r_{t+\tau} \\
& +\int_{-\infty}^{r_{L}}\left(f_{t, \tau}-r_{t+\tau}\right) \frac{1}{\omega_{\tau} \sqrt{2 \pi}} \exp \left[-\frac{1}{2}\left(\frac{r_{t+\tau}-f_{t, \tau}}{\omega_{\tau}}\right)^{2}\right] d r_{t+\tau}
\end{aligned}
$$

Define $\frac{r_{t+\tau}-f_{t, \tau}}{\omega_{\tau}}=t$ and we have $r_{t+\tau}=t \omega_{\tau}+f_{t, \tau}$. Change the range of integral to adapt the new variable $t$ and rewrite $z_{t, \tau}$. The first part of RHS of $z_{t, \tau}$ is

$$
\left(r_{L}-f_{t, \tau}\right) \int_{-\infty}^{\frac{r_{L}-f_{t, \tau}}{\omega_{\tau}}} \frac{1}{\sqrt{2 \pi}} \exp \left(-\frac{1}{2} t^{2}\right) d t=\left(r_{L}-f_{t, \tau}\right) \Phi\left(\frac{r_{L}-f_{t, \tau}}{\omega_{\tau}}\right)
$$

and the second part is

$$
\int_{-\infty}^{\frac{r_{L}-f_{t, \tau}}{\omega \tau}}\left(-t \omega_{\tau}\right) \frac{1}{\sqrt{2 \pi}} \exp \left(-\frac{1}{2} t^{2}\right) d t=-\omega_{\tau} \frac{1}{\sqrt{2 \pi}} \int_{-\infty}^{\frac{r_{L}-f_{t, \tau}}{\omega_{\tau}}} t \exp \left(-\frac{1}{2} t^{2}\right) d t
$$

\footnotetext{
${ }^{3}$ In this section, the notation with underbar _ means the restriction of the ZLB.
} 
where $\Phi\left(\frac{r_{L}-f_{t, \tau}}{\omega_{\tau}}\right)$ is the cumulative density function of standard normal distribution. Using the property of standard normal distribution, we can rewrite it as follows.

$$
\Phi\left(\frac{r_{L}-f_{t, \tau}}{\omega_{\tau}}\right)=1-\Phi\left(-\frac{r_{L}-f_{t, \tau}}{\omega_{\tau}}\right)=1-\Phi\left(\frac{f_{t, \tau}-r_{L}}{\omega_{\tau}}\right)
$$

Then calculate the second part of RHS of $z_{t, \tau}$.

$$
-\omega_{\tau} \frac{1}{\sqrt{2 \pi}} \int_{-\infty}^{\frac{r_{L}-f_{t, \tau}}{\omega_{\tau}}} t \exp \left(-\frac{1}{2} t^{2}\right) d t=\omega_{\tau} \frac{1}{\sqrt{2 \pi}} \exp \left[-\frac{1}{2}\left(\frac{r_{L}-f_{t, \tau}}{\omega_{\tau}}\right)^{2}\right]
$$

Substituting these results leads to the $z_{t, \tau}$.

$$
z_{t, \tau}=\left(r_{L}-f_{t, \tau}\right)\left[1-\Phi\left(\frac{f_{t, \tau}-r_{L}}{\omega_{\tau}}\right)\right]+\omega_{\tau} \frac{1}{\sqrt{2 \pi}} \exp \left[-\frac{1}{2}\left(\frac{r_{L}-f_{t, \tau}}{\omega_{\tau}}\right)^{2}\right]
$$

Substituting $z_{t, \tau}$ into $\underline{\mathrm{f}}_{t, \tau}=f_{t, \tau}+z_{t, \tau}$ leads to the expression of $\underline{\mathrm{f}}_{t, \tau}$.

$$
\underline{\mathrm{f}}_{t, \tau}=f_{t, \tau}+z_{t, \tau}=r_{L}+\left(f_{t, \tau}-r_{L}\right) \Phi\left(\frac{f_{t, \tau}-r_{L}}{\omega_{\tau}}\right)+\omega_{\tau} \frac{1}{\sqrt{2 \pi}} \exp \left[-\frac{1}{2}\left(\frac{f_{t, \tau}-r_{L}}{\omega_{\tau}}\right)^{2}\right]
$$

Given all results derived above, the interest rate $\underline{\mathrm{R}}_{t, \tau}$ in the shadow/ZLB-GATSM framework still has the analogous expression of the counterpart in general GATSM framework ${ }^{4}$,

$$
\underline{\mathrm{R}}_{t, \tau}=\frac{1}{\tau} \int_{0}^{\tau} \underline{\mathrm{f}}_{t, u} d u
$$

where $\underline{\mathrm{f}}_{t, u}$ has been derived in previous analysis.

\section{Data and Estimation}

In this section, we use the model derived in previous section and Japan's yield curve data to estimate this model. The results ${ }^{5}$ from estimation are parameters, estimated values of two factors and fitted values of short interest rate. Three monetary policy measures can be directly calculate from the these results, which will be introduced in next section.

\subsection{Japan Yield Curve Data}

The yield curve data set of Japan is Japanese government bond data from 1992/07/10 (Friday) to 2016/11/24 (Thursday), daily frequency of 5-business days week with 6360 observations, obtained from Bloomberg database. To reduce the burden of computation, we chose the end of month data as one observation for one month, then we get 293 observations from 1992M7 to 2016M11, monthly frequency. The maturity of yield curve is from 3-months to 30-years and 3-months interest rate is adapted to be the short interest rate $r_{t}$. Interest rates with other maturities are equivalent to be $R_{t, \tau}$ in term structure models. The summary statistics of all 12 series are given in Table 1 .

Table 1. Summary Statistics of Japan Yield Curve Data

\begin{tabular}{ccccccc}
\hline Maturity $\tau$ & $3 \mathrm{M}^{6}$ & $6 \mathrm{M}$ & $1 \mathrm{Y}$ & $2 \mathrm{Y}$ & $3 \mathrm{Y}$ & $4 \mathrm{Y}$ \\
\hline Mean & 0.458 & 0.462 & 0.508 & 0.623 & 0.762 & 0.929 \\
Med & 0.125 & 0.131 & 0.154 & 0.248 & 0.410 & 0.575 \\
Max & 3.850 & 3.810 & 3.825 & 3.883 & 4.071 & 4.469 \\
Min & -0.379 & -0.335 & -0.326 & -0.295 & -0.289 & -0.302 \\
Std. Dev. & 0.843 & 0.826 & 0.843 & 0.894 & 0.965 & 1.053 \\
\hline Obs. & 293 & 293 & 293 & 293 & 293 & 293 \\
\hline Maturity $\tau$ & $5 \mathrm{Y}$ & $7 \mathrm{Y}$ & $10 \mathrm{Y}$ & $15 \mathrm{Y}$ & $20 \mathrm{Y}$ & $30 \mathrm{Y}$ \\
\hline Mean & 1.094 & 1.393 & 1.798 & 2.123 & 2.518 & 2.678 \\
Med & 0.721 & 1.027 & 1.489 & 1.833 & 2.188 & 2.451 \\
Max & 4.714 & 5.311 & 5.392 & 6.020 & 6.205 & 6.090 \\
Min & -0.305 & -0.309 & -0.216 & -0.078 & 0.078 & 0.138 \\
Std. Dev. & 1.122 & 1.213 & 1.252 & 1.291 & 1.261 & 1.161 \\
\hline Obs. & 293 & 293 & 293 & 293 & 293 & 293 \\
\hline
\end{tabular}

\footnotetext{
${ }^{4}$ This expression does not have analytic solution in closed-form and need to be evaluated numerically

${ }^{5}$ Estimation results are available upon request.
} 
We can find that with the increasing of maturity, the standard deviation also increases, which means the increasing of uncertainty of long maturity. Note that the minimum values of yield curve data from 3-months to 15-years are all negative. The time series plot of yield curve data is given in Figure 1.

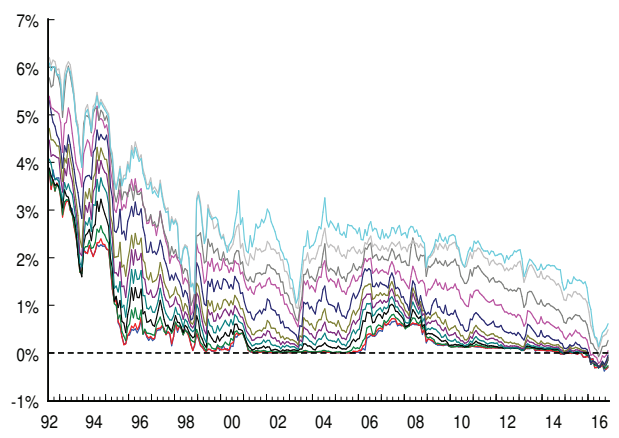

(a) Japan Government Bond Yield Curve Data

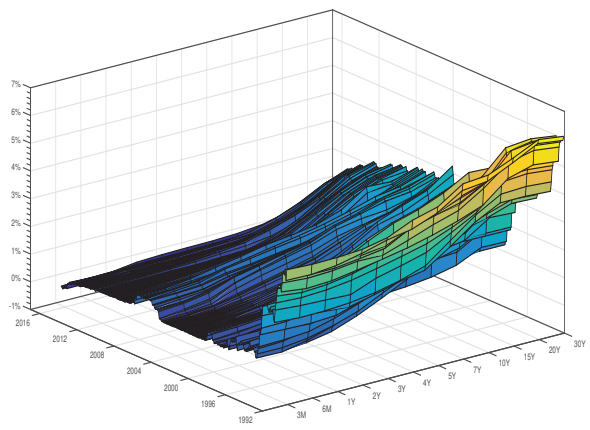

(b) Japan Government Bond Yield Curve Data (3D view)

Figure 1. Japan Government Bond Yield Curve Data

From the 3D view of yield curve data, we can confirm that the yield curves of all maturities have shifted down since 1999. Figure 2 shows the plot of 3-months interest rate of Japan government bond and un-collateralized overnight call rate, which is generally recognized as the official short policy interest rate of Bank of Japan (BoJ). We can find that after 1999, these two kinds of typical short interest rates have been evolving near the ZLB. Although during the ZLB period, BoJ has adopted several unconventional monetary policy programs and during the same period, the macroeconomy in Japan also has dramatic fluctuations, we can't get further information from the short interest rate if we still consider it as the measure of monetary policy. This is why we need shadow/ZLB-GATSM to model the short interest rate in the ZLB environment.

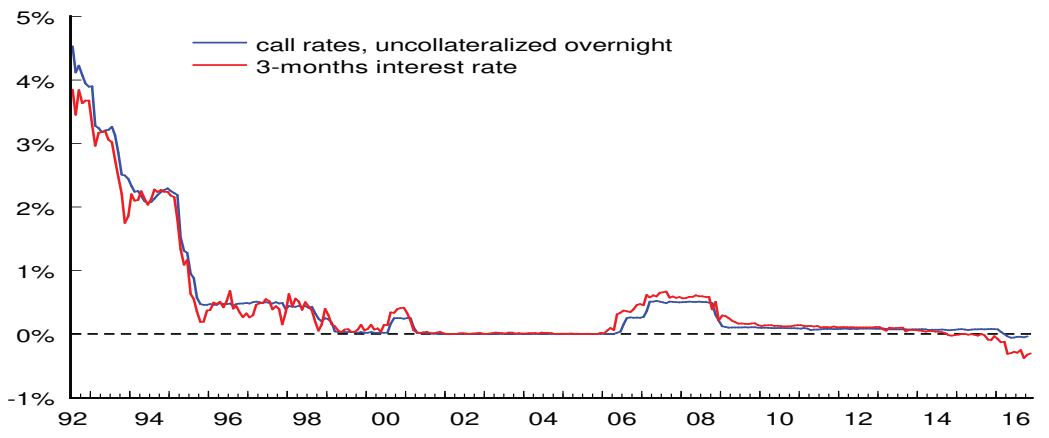

Figure 2. Short Interest Rates in Japan

Note that money statistics and long-maturity interest rates can be potentially used to quantify the stance of monetary policy in the ZLB environment. But there are some fatal defects of them. For money statistics such as money supply or money growth, history has told us that the relationships between money statistics and macroeconomic variables haven't been reliable since 1980s, especially in developed economies. For long-maturity interest rates such as 10-years interest rate of government bond, even long-maturity interest rates are not constrained by the ZLB and there exists research that supports the view that the long-maturity interest rates can response to monetary policy events, the fluctuations in long-maturity interest rates can be the results of other factors such as neutral interest rates, inflation expectations and so on. In another word, long-maturity interest rates are noisy measures of stance of monetary policy. Also, the amount of large-scale asset purchase can be used as the measure of stimulative degree of monetary easing, but from the results of this paper, the first round of monetary easing from $2001 \mathrm{M} 3$ to $2006 \mathrm{M} 3$, the following round from 2010M10 to 2013M3 and the latest QQE from 2013M4, the estimated stimulative degree of monetary policy doesn't have much difference even the scale of asset purchase is quite different during different policy programs.

\footnotetext{
${ }^{6} \mathrm{M}=$ Month and $\mathrm{Y}=$ Year.
} 


\subsection{Estimation}

The estimation period is from $1992 \mathrm{M} 7$ to $2016 \mathrm{M}^{7}$. GATSM is generally estimated in state-space form with Kalman filter and maximum likelihood method. Compared to general GATSM, the framework proposed by Krippner and its specified implementation K-ANSM(2) have nonlinear functions of state variables $x_{t}$ via the CDF or PDF of normal distribution. Iterated Extended Kalman Filter (henceforward IEKF) can handle the nonlinearity. For technical details of IEKF, please refer to Krippner (2015, pp.117-126) which provides the instruction of IEKF in the estimation of shadow/ZLB term structure model. Wu and Xia (2016), Bauer and Rudebusch (2016) also used the same algorithm.

The K-ANSM(2) has 11 parameters to estimate. Denote the set of all estimated parameters as $\Omega=\left\{r_{L}, \varphi, \theta_{1}, \theta_{2}, \kappa_{11}, \kappa_{12}, \kappa_{21}\right.$, $\left.\kappa_{22}, \sigma_{1}, \sigma_{2}, \rho_{12}\right\}$. Here we consider the lower bound of nominal interest rate $r_{L}$ as a parameter to be estimated instead of setting $r_{L}=0$ di rectly. The estimation can be conducted by using the Matlab code ${ }^{8}$ provided by Krippner (2015). The state equation for K-ANSM(2) under objective $\mathbb{P}$ measure is given in discrete time where $\Delta t$ is time span between two observations.

$$
x_{t}=\theta^{\mathbb{P}}+e^{-\kappa^{\mathbb{P}} \Delta t}\left(x_{t-1}-\theta^{\mathbb{P}}\right)+\varepsilon_{t}
$$

Variance of innovation $\varepsilon_{t}$ is calculated as follows.

$$
\mathbb{V} \mathbb{R}^{\mathbb{P}}\left[\varepsilon_{t}\right]=\int_{0}^{\Delta t} e^{-\kappa^{\mathbb{P}} u} \sigma \sigma^{\top} e^{-\left(\kappa^{\mathbb{P}}\right)^{\top} u} d u
$$

Note that $\underline{\mathrm{R}}_{t, \tau}=\frac{1}{\tau} \int_{0}^{\tau} \underline{\mathrm{f}}_{t, u} d u$ is a function of state variables $x_{t}$, maturity $\tau$ and other parameters, so measurement equation for K-ANSM(2) can be represented as

$$
\underline{\mathrm{R}}_{t, \tau}=\underline{\mathrm{R}}\left(x_{t}, \tau, \Omega\right)+\eta_{t}
$$

where $\eta_{t}$ is a $12 \times 1$ vector of measurement errors ${ }^{9}$. The variance of measurement errors which is a $12 \times 12$ diagonal matrix $\mathbb{V} \mathbb{R}\left(\eta_{t}\right)=\operatorname{diag}\left[\sigma_{\eta}^{2}, \cdots, \sigma_{\eta}^{2}\right]$ are assumed to have homoscedasticity $\sigma_{\eta}^{2}$ and to be independent to each other. $\varepsilon_{t}$ in state equation and $\eta_{t}$ in measurement equation are also assumed to be uncorrelated to each other.

\subsection{Results}

We conducted estimation using the monthly frequency data which were extracted from the original daily frequency data by choosing end-monthly observation of yield curve. Monthly estimates are used to match other data which is only available in monthly frequency such as un-collateralized overnight call rate or Consumer Price Index (CPI). From Table 2, we can find that $r_{L}$ is a very small number, approximately equal to zero.

Table 2. Estimated Parameters of K-ANSM(2)

\begin{tabular}{ccc}
\hline \multicolumn{3}{c}{ Monthly Estimation } \\
\hline Parameters & Estimate & Std.Error \\
\hline$r_{L}$ & 0.0006 & 0.0001 \\
$\varphi$ & 0.1295 & 0.0033 \\
$\kappa_{11}$ & 0.0614 & 0.0060 \\
$\kappa_{12}$ & 0.0101 & 0.0008 \\
$\kappa_{21}$ & 0.0410 & 0.0107 \\
$\kappa_{22}$ & 0.0072 & 0.0009 \\
$\theta_{1}$ & 0.0741 & 0.0098 \\
$\theta_{2}$ & -0.3554 & 0.0224 \\
$\sigma_{1}$ & 0.0119 & 0.0004 \\
$\sigma_{2}$ & 0.0133 & 0.0007 \\
$\rho_{12}$ & -0.8920 & 0.0134 \\
\hline
\end{tabular}

\footnotetext{
${ }^{7}$ As mentioned in the Section 1, the data from 2016M2 is negative and inapplicable to the computation of model estimation

${ }^{8}$ The Matlab code for estimation is available from homepage of Krippner in the website of Reserve Bank of New Zealand.

${ }^{9}$ Because we have 12 series yield curve data with different maturities, we have to specify 12 measurement equations.
} 


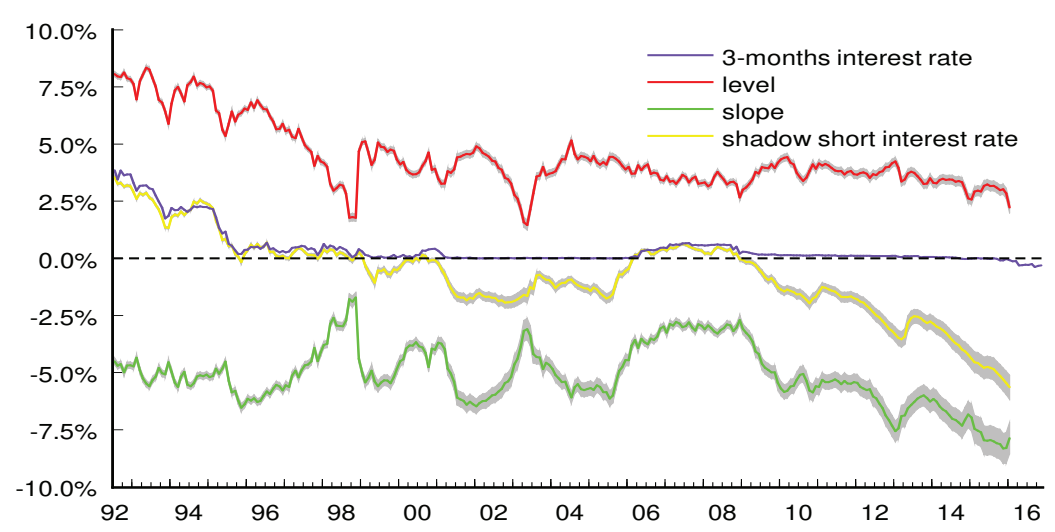

Figure 3. Results from K-ANSM(2)

Estimated two state variables, shadow short interest rate are plotted in Figure 3. The gray shaded area between lines of point estimates is the range of plus/minus one estimated standard error. During the non-ZLB period, the shadow interest rate has almost same path as actual short interest rate does during non-ZLB period. But during the ZLB period, short interest rate remains static near the ZLB and the shadow short interest rate still evolves to negative values. Given these estimated series from K-ANSM(2), we put them in a time line of monetary policy events and then evaluate the stance of monetary policy in Japan.

\section{Measures of Monetary Policy in Japan}

Three quantitative measures can be calculated from K-ANSM(2) and then can be used as quantitative indicators of the stance of monetary policy. We explain these indicators one by one in Section 4 in a context of monetary policy events.

\subsection{Shadow Short Interest Rate}

The concept of shadow short interest rate (SSR) was firstly proposed by Black (1995). If the ZLB wouldn't exist and nominal interest rate could decrease to negative value freely, the economic agents would hold physical currency rather than invest in government bonds. The value of this call option that the economic agents could choose to hold currency in hand with zero return plus the SSR is equal to zero theoretically. In another word, the call option of holding currency would have positive value when the economic agents expect the deep decrease of interest rates in future.

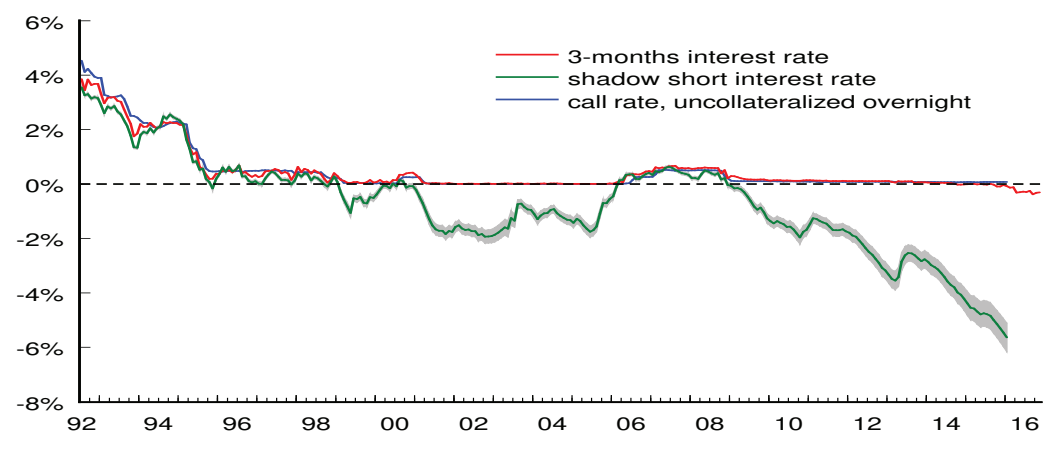

Figure 4. Shadow Short Interest Rate

As we have already mentioned previously, the SSR is the shortest maturity interest rate from the estimated shadow yield curve, which can take negative values in the ZLB environment. We can confirm from Figure 4 that the SSR is approximately equal to the 3-months interest rate and policy rate during the non-ZLB period. Note that the estimated results from this paper are similar to Ichiue and Ueno (2013) or Imakubo and Nakajima (2015). Table 3 shows the major monetary policy regimes officially announced by BoJ since 1999 where each green shade indicates the period of each policy regime. But we can at least confirm the negative SSR levels are correlated with the evolution of monetary policy 
events. Although the SSR shows different response to each policy event, the main trend of the SSR is the decrease with the evolution of unconventional monetary policy from zero interest rate policy to QQE in Japan.

Table 3. Monetary Policy Regimes of BoJ

\begin{tabular}{ll}
\hline & The Policy Evolution of BoJ since 1999 \\
\hline 1999-Feb-2 to 2000-Aug-11 & Zero Interest Rate Policy (pink shaded area in Figure 5) \\
\hline 2001-Mar-19 to 2006-Mar-9 & Quantitative Easing (yellow shaded area in Figure 5) \\
\hline 2010-Oct-5 to 2013-Mar-20 & Comprehensive Monetary Easing (green shaded area in Figure 5) \\
\hline 2013-Apr-4 to 2016-Jan-29 & Price Stability Target of 2\% and Quantitative and Qualitative Monetary Easing (blue shaded area in Figure 5) \\
\hline 2016-Feb-16 to 2016-Sep-19 & Price Stability Target of 2\% and Quantitative and Qualitative Monetary Easing with a Negative Interest Rate \\
\hline
\end{tabular}

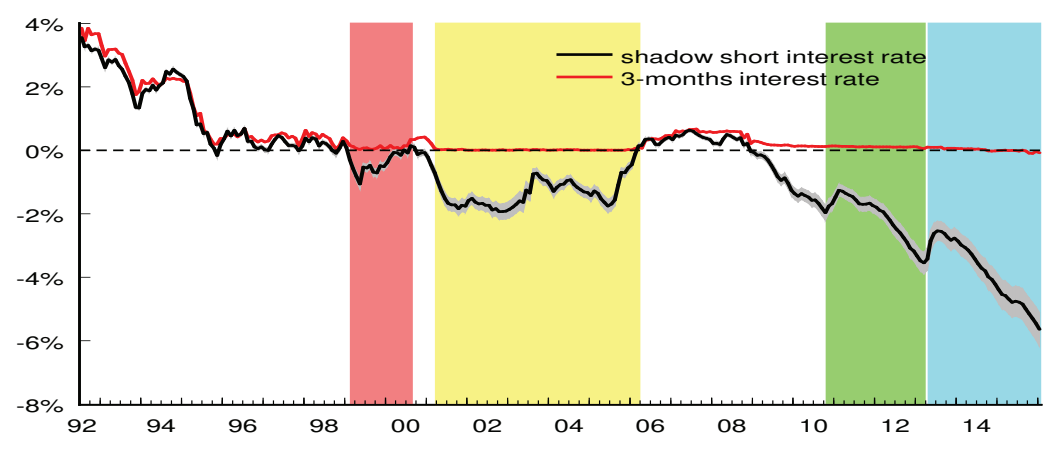

Figure 5. Shadow Short Interest Rate in Different Monetary Policy Regimes

Note that it is better to consider the SSR as an ordinal measure of monetary policy. The SSR can be used to track the unconventional monetary policy events in a consistent way. Generally, more lower values are consistent with more monetary stimulus, and vice versa. But in real world, the economic agents can't transact with negative nominal interest rates, the change of the SSR doesn't mean that it can have real effect to economy in the same way as the change of policy rate in the conventional non-ZLB environment.

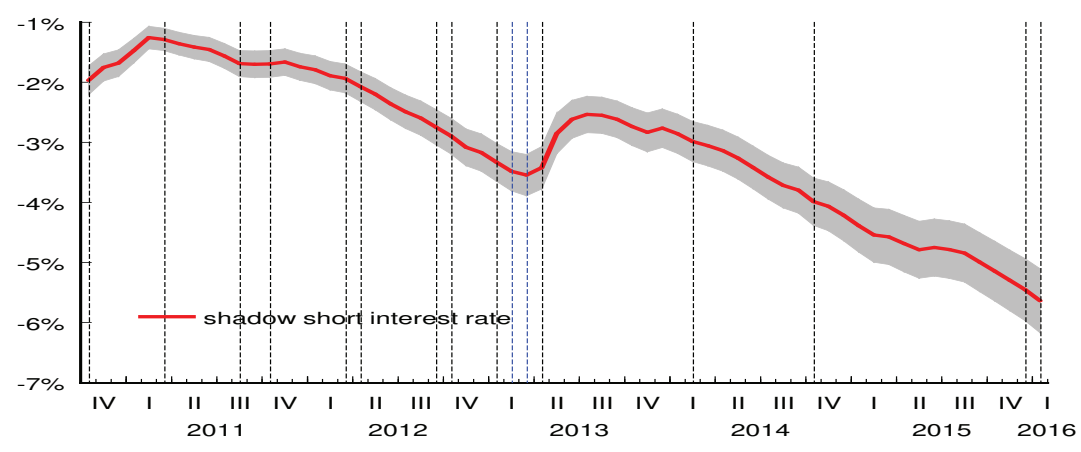

Figure 6. Monetary Policy Events from 2010M10 to 2016M1

Figure 6 is a close-up of Figure 5, zooming up from 2010M10, the start of Comprehensive Monetary Easing to 2016M1, the end of first phase of Quantitative and Qualitative Monetary Easing. The vertical dashed lines plotted in Figure 6 are the indicators for the major policy decisions made by policy board of BoJ. For most of this period, the policy decisions are the "maintain the status quo" or "additional monetary easing", and the shadow short interest rate shows response to the policy decisions, decreasing with more negative values. We located two policy decisions which are "send-off of additional monetary easing" by two blue dashed lines at 2013/2/14 and 2013/3/7. The shadow short interest rate increased after these two decisions in a consistent way of short interest rate in general environment. But during the same period, call rate and 3-months interest rate all didn't have much response to the policy changes and we can't get any information from these 
general monetary policy indicators. This is why we should use the shadow short interest rate as a proxy of policy rate in the ZLB environment.

\subsection{Expected Time to Zero}

Figure 7 shows the another monetary policy measure in the ZLB environment, Expected Time to Zero (ETZ). According to Krippner (2015), ETZ can provide an implied market-based expectation of when the actual short interest rate is expected to rise from zero and return to the value of the non-ZLB environment. Note that from Section 2, the expected path of shadow short interest rate is $\mathbb{E}_{t}^{\mathbb{Q}}\left(r_{t+\tau_{0}}\right)=L_{t}+e^{-\varphi \tau_{0}} S_{t}=0$ where the $\tau_{0}$ is $\mathrm{ETZ}_{t}=\tau_{0}$ and can be calculated from the estimated values of state variables $L_{t}, S_{t}$ and parameter $\varphi$ by solving the equation as $\tau_{0}=-\frac{1}{\varphi} \ln \left(-\frac{L_{t}}{S_{t}}\right)$.

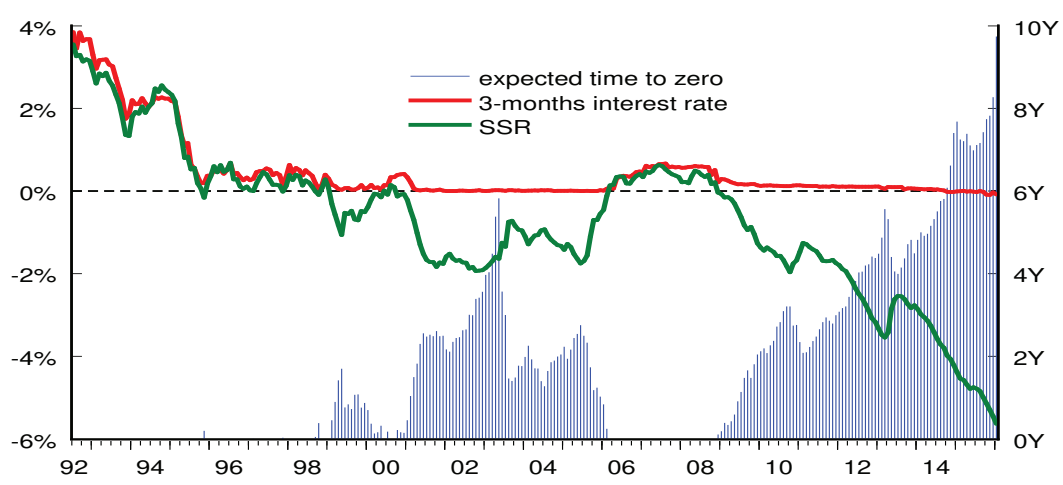

(a) Expected Time to Zero from 1992M7 to 2016M1

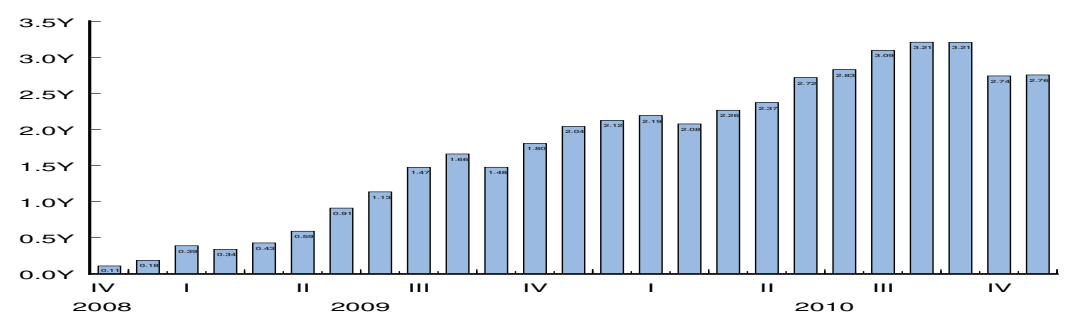

(b) Expected Time to Zero from 2008M12 to 2010M12

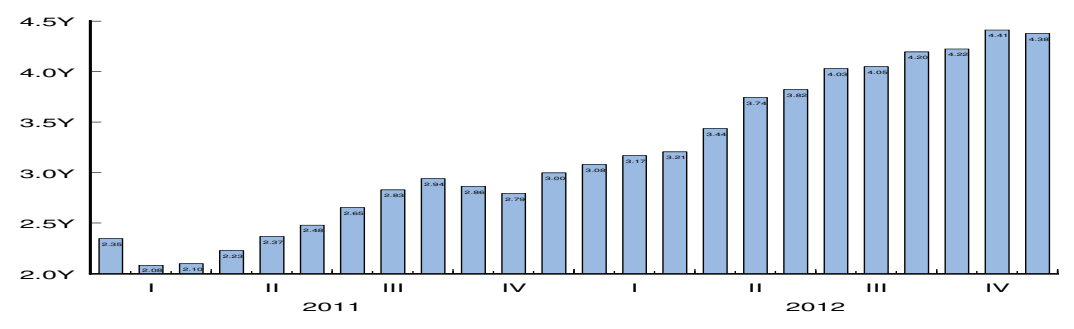

(c) Expected Time to Zero from 2011M1 to 2012M12

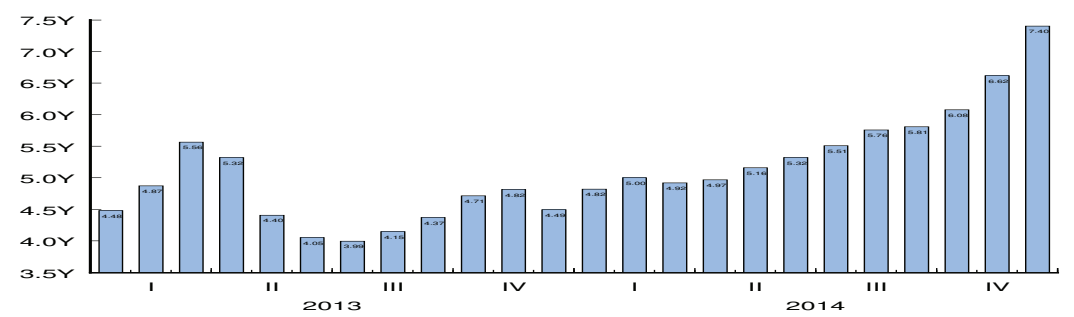

(d) Expected Time to Zero from 20013M1 to 2014M12 


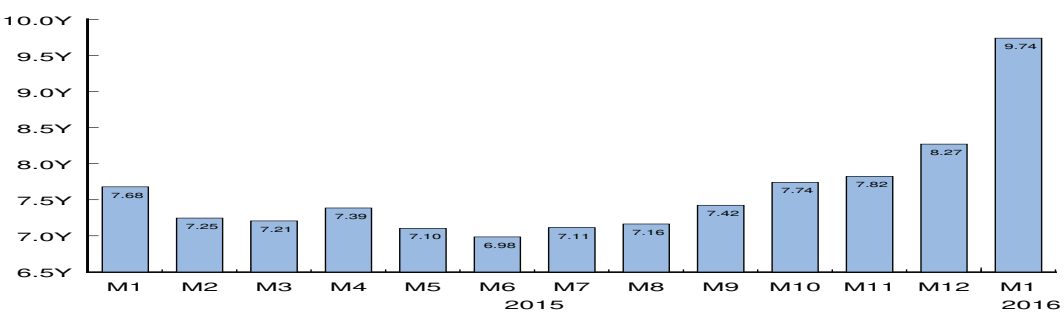

(e) Expected Time to Zero from 2015M1 to 2016M1

Figure 7. Expected Time to Zero

Figure 7 provides the ETZ (right axis) in Japan. During the Zero Interest Rate Policy period, Qualitative Easing period and Comprehensive Monetary Easing period, the ETZ shows consistent path with the evolution of monetary policy, especially the start and end of each policy. Since the QQE is still ongoing, the ETZ is a good measure providing the information about how long economic agents are likely to face the ZLB and when and how the central bank should consider the exit strategy or forward guidance. For example, at the end point of the sample, 2016M1, the ETZ is almost 10 years. Adding this expected horizon of the ZLB environment provides a range from 2016M1 to 2026M1. This range may be the reference for BOJ's forward guidance about the time point of the end of the ZLB environment. Though BOJ doesn't officially announce this the time point of the end of the ZLB environment, the ETZ can still provide the market-implied expectation about how long the ZLB will continue. Since there doesn't exist market survey about this expectation, the ETZ is the only available measure if we want to know it. From Figure 7, we can imply that the ZLB environment in Japan will last for quite long time, especially from 2013M4, the start of first phase of QQE. There exists an obvious trend that the ETZ will increase with the deepening of QQE.

\subsection{Effective Monetary Stimulus}

Before we introduce the concept of Effective Monetary Stimulus (EMS), we consider the neutral interest rate of economy. The neutral interest rate is the short interest rate when the economy will achieve its long-run equilibrium level, the balance of investment and saving. If policy rate is equal to the neutral interest rate, the monetary policy is neutral to economy, neither easy and stimulative nor tight and suppressive. In the non-ZLB environment, if the policy rate is below the neutral rate with an expectation that the policy rate will finally revert to the neutral rate level as time evolves, the EMS can be calculated as the area between the expected path of the short interest rate and the neutral interest rate. In the ZLB environment, the explicit stance of monetary policy is a zero interest rate policy but with the expectation that the short interest rate will rise to normal positive level at some horizon in the future and finally return to its neutral level. The EMS has consistent interpretation in both non-ZLB and ZLB environment.

Now we explain the EMS in K-ANSM(2). We have already showed that the short interest rate $r_{t}=L_{t}+S_{t}$ has its expected path $\mathbb{E}_{t}^{\mathbb{Q}}\left(r_{t+\tau} \mid x_{t}\right)=L_{t}+e^{-\varphi \tau} S_{t}$ in Section 2.3. For the long-horizon $\tau \rightarrow \infty$, the expectation of the shadow short interest rate is $\lim _{\tau \rightarrow \infty} \mathbb{E}_{t}^{\mathbb{Q}}\left(r_{t+\tau} \mid x_{t}\right)=L_{t}$ and the expected path of short interest rate relative to its long-run expected value is

$$
\lim _{\tau \rightarrow \infty} \mathbb{E}_{t}^{\mathbb{Q}}\left(r_{t+\tau} \mid x_{t}\right)-\max \left\{0, \mathbb{E}_{t}^{\mathbb{Q}}\left(r_{t+\tau} \mid x_{t}\right)\right\}=\left\{\begin{array}{l}
L_{t}, \text { if } \mathbb{E}_{t}^{\mathbb{Q}}\left(r_{t+\tau} \mid x_{t}\right)<0 \\
-e^{-\varphi \tau} S_{t}, \text { if } \mathbb{E}_{t}^{\mathbb{Q}}\left(r_{t+\tau} \mid x_{t}\right) \geq 0
\end{array}\right.
$$

where $L_{t}$ is the estimate of the neutral interest rate at time $t$. The stance of monetary policy can be identified from this policy/neutral interest rate gap. The EMS is the area that can be calculated from the integral of this gap with respect to $\tau$ with a range $(0, \infty)$.

$$
E M S_{t}=\int_{0}^{\infty}\left(L_{t}-\max \left\{0, \mathbb{E}_{t}^{\mathbb{Q}}\left(r_{t+\tau} \mid x_{t}\right)\right\}\right) d \tau
$$

If $\mathbb{E}_{t}^{\mathbb{Q}}\left(r_{t+\tau} \mid x_{t}\right) \geq 0$ at all horizons $\tau \in(0, \infty)$, the EMS is $E M S_{t}=\int_{0}^{\infty}\left(-e^{-\varphi \tau} S_{t}\right) d \tau=-\frac{S_{t}}{\varphi}$ and if $\mathbb{E}_{t}^{\mathbb{Q}}\left(r_{t+\tau} \mid x_{t}\right)<0$, for $\mathbb{E}_{t}^{\mathbb{Q}}\left(r_{t+\tau} \mid x_{t}\right)$ is a monotonic function of horizon $\tau$, if $\mathbb{E}_{t}^{\mathbb{Q}}\left(r_{t+\tau} \mid x_{t}\right)$ has an intersection with zero at horizon $\tau_{0}$, it has only one intersection and $\mathbb{E}_{t}^{\mathbb{Q}}\left(r_{t+\tau} \mid x_{t}\right)<0$ holds for values of $\tau<\tau_{0}$ when $r_{t}=S_{t}+L_{t}<0 . \tau_{0}$ can be solved as follows by setting $\mathbb{E}_{t}^{\mathbb{Q}}\left(r_{t+\tau} \mid x_{t}\right)=0$.

$$
L_{t}=-S_{t} e^{-\varphi \tau_{0}} \Rightarrow \tau_{0}=-\frac{1}{\varphi} \log \left(-\frac{L_{t}}{S_{t}}\right)
$$


The EMS in this case is given by following integral.

$$
E M S_{t}=\int_{0}^{\tau_{0}} L_{t} d \tau+\int_{\tau_{0}}^{\infty}\left(-S_{t} e^{-\varphi \tau}\right) d \tau=\tau_{0} L_{t}-\frac{S_{t}}{\varphi} e^{-\varphi \tau_{0}}
$$

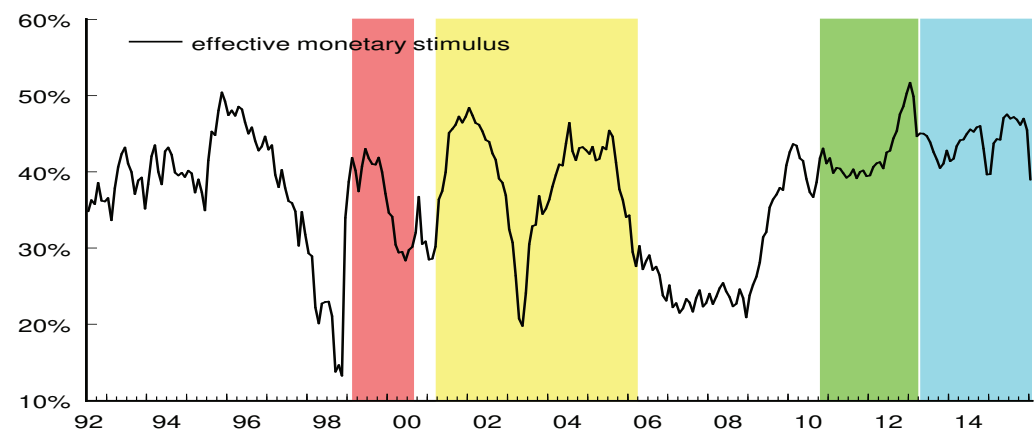

Figure 8. Effective Monetary Stimulus

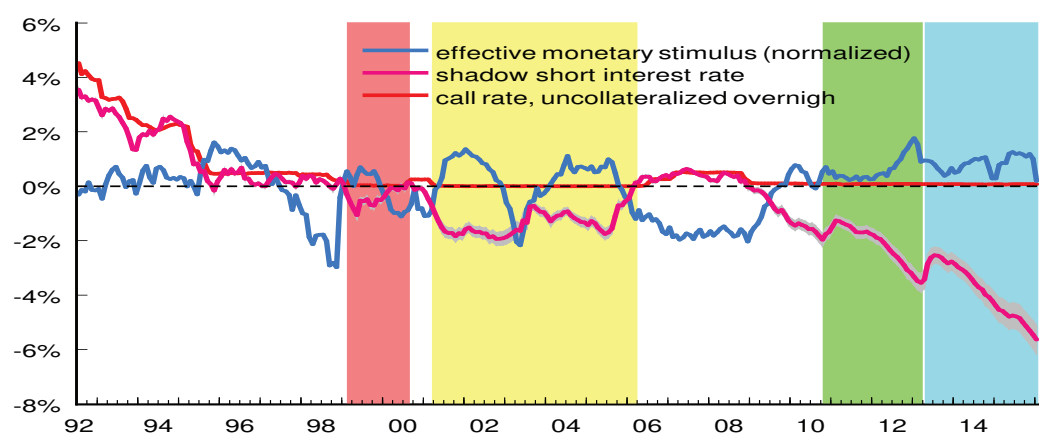

Figure 9. Normalized Effective Monetary Stimulus

Figure 8 shows the monthly estimate of EMS. For a intuitive view of the EMS, we normalized the monthly estimate of EMS by $\frac{\mathrm{EMS}_{t}-\text { mean }\left(\mathrm{EMS}_{t}\right)}{\left.\text { std.er(EMS } \mathrm{E}_{t}\right)}$ and plot the SSR and call rate together in Figure 9, we can find that the SSR and EMS have a consistent relationship in the ZLB environment, lower SSR coincides with higher EMS, just like that the policy rate and EMS have in the non-ZLB environment. When SSR rises, the EMS decreases and vice versa. The estimated EMS from K-ANSM(2) shows very good traceability of monetary policy in Japan. We also calculated the mean of EMS in different schemes of monetary policy. The mean of EMS in QE (and comprehensive monetary easing) from $2001 \mathrm{M} 3$ to $2013 \mathrm{M} 3$ is $36 \%$ and the mean of EMS in QQE from 2013M4 to 2016M1 is 44\%, which shows the QQE is more aggressive than the previous QE, but only $8 \%$ of increasing. We can conclude that both EMS and ETZ show very accurate and consistent traceability of monetary policy.

\subsection{Relationship between EMS and Macroeconomic Variables}

In previous analysis, we have already found that the EMS and SSR have consistent traceability of monetary policy in the ZLB environment. In Figure 10, we check relationship between the macroeconomic variables and EMS. From Figure 10, we can find that the relative low EMS coincides with deterioration of GDP gap ${ }^{10}$. BoJ's unconventional monetary policy from 2001 to 2006, we can find that the GDP gap had achieved improvement. After the QE, the EMS decreased to relative low level before the global financial crisis. After 2008's financial crisis, the EMS begun to increase with the improvements of GDP gap. The increasing of the EMS which represents the new rounds of monetary easing policy leads to the improvement of GDP gap, which can be confirmed from the figure. We also estimated a simple equation to check the whether the degree of monetary policy measured by EMS can lead to the change of the macroeconomic variables.

\footnotetext{
${ }^{10}$ Please see https://www.boj.or.jp/research/research_data/gap.htm/.
} 


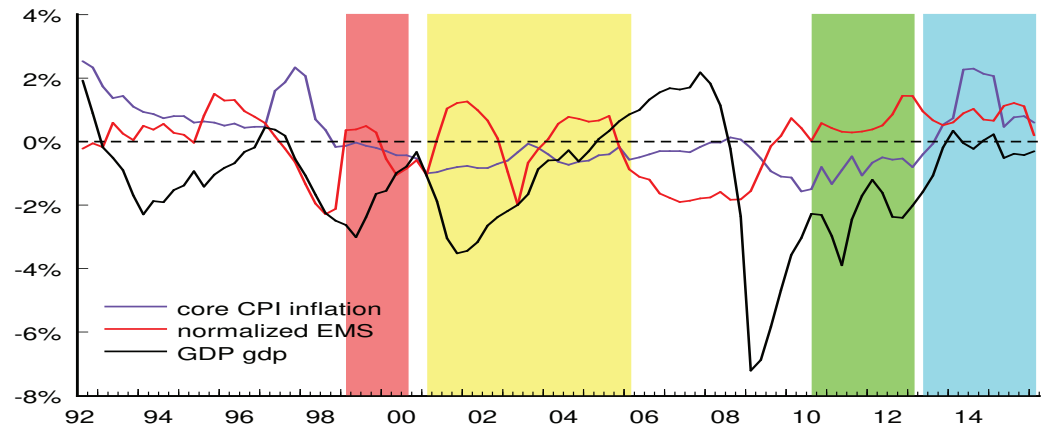

Figure 10. Normalized EMS, Core CPI inflation and GDP Gap

As the results of estimation showed in Table 4, from 2010Q3 to 2016Q1, the stimulative degree of monetary policy represented by EMS has a positive effect on GDP gap. 1\% decreasing of the EMS leads to $0.88 \%$ decreasing of GDP gap. We also tried the regression of EMS and $\mathrm{CPI}^{11}$, but did't get a statistical significant result. This may imply that the monetary easing in Japan has limited effect on pushing up the price level.

Table 4. Normalized EMS and GDP Gap $\left(^{* * *} p\right.$-value $\left.<0.01\right)$

\begin{tabular}{cccc}
\hline \multicolumn{4}{c}{$Y_{t}=\beta_{0}+\beta_{1} Y_{t-1}+\beta_{2} E_{t-3}+\varepsilon_{t}$} \\
\hline & Coef. & Std.Error & t-Statistic \\
$\beta_{0}$ & $-0.836^{* * *}$ & 0.296 & -2.824 \\
$\beta_{1}$ & $0.728^{* * *}$ & 0.089 & 8.156 \\
$\beta_{2}$ & $0.880^{* * *}$ & 0.308 & 2.851 \\
\hline \multicolumn{2}{c}{$R^{2}=0.83$} & Sample: $2010 \mathrm{Q} 32016 \mathrm{Q} 1$ \\
\hline \multicolumn{3}{l}{$Y_{t}=$ GDP Gap } & \multicolumn{2}{c}{$E_{t}=$ Normalized EMS } \\
\hline
\end{tabular}

\section{Concluding Remarks}

In this paper, we estimated the K-ANSM(2) model, a shadow/ZLB term structure model that can be used to model short interest rate in the ZLB environment. We can derive three monetary policy measures from this model which can provide the consistent view of the stance of monetary policy in Japan. The SSR acts as a proxy of general short interest rate in the ZLB environment. We can find that the SSR has already decreased to $-6 \%$, which means that the value of holding physical currency is $+6 \%$. The ETZ shows that the expected horizon of the ZLB in Japan will last for at least 8 years at the end of 2015. As far as we are concerned, this paper firstly provided the answer about how long the ZLB will last in Japan because there doesn't exist any research or survey about this expectation of the market. The EMS provides a consistent way to track the stance of monetary policy in Japan. Generally, lower SSR means further stimulus of monetary policy, but from the Figure 9, we can find that the peak stimulus of QQE is not stronger than the first time of monetary easing conducted by BoJ from 2001 to 2006, but the mean of EMS in QQE is still 8\% larger than the mean of EMS in previous scheme of policy. This may imply that the monetary easing policy, large-scale purchasing of the government bond and increasing money supply, is more aggressive than before, but approaching its limit, no matter how many government bond have been purchased, the whole volume of government bond is finite and it is not far from the limit of QQE. We found that the stimulative degree of monetary policy represented by EMS has a positive effect $(+0.88 \%)$ on GDP gap from $2010 \mathrm{Q} 3$ to 2016Q1, but doesn't have significant effect to inflation in a similar regression. Note that we have already confirmed that the SSR and EMS can be used as measures of monetary policy, these measures can be directly used in other econometric procedures to evaluate the effects of monetary policy. This paper provides purely empirical results from a K-ANSM(2) model and we will use the results from this paper for further empirical applications.

\section{References}

Bauer, M. D., \& Rudebusch, G. D. (2016). Monetary Policy Expectations at the Zero Lower Bound. Journal of Money, Credit and Banking, 48(7), 1439-1465.

Black, F. (1995). Interest Rates as Options. The Journal of Finance, 50(5), 1371-1376.

\footnotetext{
${ }^{11}$ Please see http://www.stat.go.jp/data/cpi/.
} 
Heath, D., Jarrow, R., \& Morton, A. (1992). Bond Pricing and the Term Structure of Interest Rates: A New Methodology for Contingent Claims Valuation. Econometrica: Journal of the Econometric Society, 77-105.

Ichiue, H., \& Ueno, Y. (2013). Estimating Term Premia at the Zero Bound: An Analysis of Japanese, US, and UK Yields (No. 13-E-8). Bank of Japan.

Imakubo, K., \& Nakajima, J. (2015). Estimating Inflation Risk Premia from Nominal and Real Yield Curves using A Shadow-Rate Model (No. 15-E-1). Bank of Japan.

Kim, D. H., \& Singleton, K. J. (2012). Term Structure Models and the Zero Bound: An Empirical Investigation of Japanese Yields. Journal of Econometrics, 170(1), 32-49.

Krippner, L. (2015). Zero Lower Bound Term Structure Modeling: A Practitioners Guide. Springer.

Nelson, C. R., \& Siegel, A. F. (1987). Parsimonious Modeling of Yield Curves. Journal of Business, 473-489.

Wu, J. C., \& Xia, F. D. (2016). Measuring the Macroeconomic Impact of Monetary Policy at the Zero Lower Bound. Journal of Money, Credit and Banking, 48(2-3), 253-291.

\section{Copyrights}

Copyright for this article is retained by the author(s), with first publication rights granted to the journal. This is an open-access article distributed under the terms and conditions of the Creative Commons Attribution license which permits unrestricted use, distribution, and reproduction in any medium, provided the original work is properly cited. 